\title{
Pesticides Registered for Tropical Fruit Crops in Florida ${ }^{1}$
}

\section{Jonathan H. Crane and Mark A. Mossler ${ }^{2}$ \\ Introduction}

Please note, this list may not cite all chemical and common names of pesticides legally registered at the present time. This list does not constitute endorsement of any pesticide chemical named; these pesticides may be available under other names locally. Some pesticides require a license in order to buy and/or apply. Always follow the pesticide label instructions. Please note the superscript adjacent to some brand names. Some pesticides are for 1) non-bearing trees only; 2) non-bearing trees in the nursery only and; 3 ) for use on dormant trees only. Please contact your local Agriculture or Horticulture Extension Agent if you have any questions.

\section{List of tables.}

Table 1. Atemoya and sugar apple.

Table 2. Avocado.

Table 3. Banana and plantain.

Table 4. Barbados cherry.

Table 5. Black sapote.
Table 6. Carambola.

Table 7. Coconut.

Table 8. Guava.

Table 9. Jackfruit.

Table 10. 'Tahiti' lime, Key lime, kumquat, and pummelo.

Table 11. Lychee and longan.

Table 12. Mamey sapote.

Table 13. Mango.

Table 14. Papaya.

Table 15. Passion fruit.

Table 16. Sapodilla.

Table 17. Wax jambu.

Table 18. White sapote.

1. This document is Fact Sheet HS177, one of a series of the Horticultural Sciences Department, Florida Cooperative Extension Service, Institute of Food and Agricultural Sciences, University of Florida. New publication, May 2003. Please visit the FAIRS Web site at http://edis.ifas.ufl.edu.

2. J.H. Crane, professor and tropical fruit crops extension specialist, Tropical Research and Education Center, Homestead, FL; M.A. Mossler, assistant in, pest management information, Food Science and Human Nutrition Department, Cooperative Extension Service, Institute of Food and Agricultural Sciences, University of Florida, Gainesville, 32611. 
Table 1. Pesticides registered for atemoya and sugar apple in Florida.

\begin{tabular}{|c|c|c|c|}
\hline Class & Chemical name & Brand name(s) & Pest(s) controlled \\
\hline \multirow[t]{19}{*}{ Insecticide } & pyrethrin + rotenone & Pyrellin (atemoya only) & $\begin{array}{l}\text { aphids, lepidoptera, } \\
\text { thrips }\end{array}$ \\
\hline & potassium salts of fatty acids & Safer Soap & $\begin{array}{l}\text { aphids, lace bug, plant } \\
\text { bugs, mites, thrips, } \\
\text { scales }\end{array}$ \\
\hline & various refined horticultural oils & $\begin{array}{l}\text { Sunspray, citrus spray oil, crop oil, } \\
\text { FC 435-66, FC 455-88, others }\end{array}$ & aphids, mites, scales \\
\hline & azadirachtin & Align, Azatin & general insecticide \\
\hline & spinosad & Spintor & lepidoptera \\
\hline & methidathion & Supracide & scales \\
\hline & pyrethrins & Pyronyl (atemoya only) & aphids, thrips \\
\hline & Beauveria bassiana & Mycotrol & $\begin{array}{l}\text { leaf hoppers, thrips, } \\
\text { others }\end{array}$ \\
\hline & Bacillus thuringiensis & Dipel, others & lepidoptera \\
\hline & fenoxycarb & Precision $^{1}$ & ants \\
\hline & pymetrozine & Endeavor $^{2}$ & whiteflies, aphids, \\
\hline & kaolin (clay) & Surround & $\begin{array}{l}\text { barrier and irritant to } \\
\text { various insects }\end{array}$ \\
\hline & S-methoprene & Extinguish & ants \\
\hline & hydramethylnon & Amdro $^{1}$ & ants \\
\hline & pyriproxyfen & Knack, Distance $^{2}$ & scales, thrips, ants \\
\hline & bifenazate & Floramite $^{1}$ & mites \\
\hline & fenpropathrin & Tame $^{2}$ & various insects, mites \\
\hline & hexythiazox & Savey $^{2}$ & various insects, mites \\
\hline & bifenthrin & Talstar $^{1}$ & various insects, mites \\
\hline \multirow[t]{4}{*}{ Fungicide } & azoxystrobin & Abound & $\begin{array}{l}\text { anthracnose, rust, } \\
\text { Cercospora leaf spot, } \\
\text { powdery mildew }\end{array}$ \\
\hline & copper & Kocide, others & $\begin{array}{l}\text { anthracnose, various } \\
\text { other diseases }\end{array}$ \\
\hline & mefenoxam & Subdue $\operatorname{Maxx}^{2}$ & Pythium root rot \\
\hline & Trichoderma harzianum & Rootshield ${ }^{2}$ & $\begin{array}{l}\text { root system disease } \\
\text { protectent }\end{array}$ \\
\hline \multirow[t]{3}{*}{ Herbicide } & glyphosate (systemic) & Roundup, Glyphosate & numerous weeds \\
\hline & oxyfluorfen & Goal (sugar apple only) & broadleaf weeds \\
\hline & pelargonic acid & Scythe & $\begin{array}{l}\text { broad leaf and grass } \\
\text { weeds }\end{array}$ \\
\hline Repellent & capsaicin & Deer Off & $\begin{array}{l}\text { Deer, rabbit, mouse } \\
\text { repellent }\end{array}$ \\
\hline \multicolumn{4}{|c|}{$\begin{array}{l}{ }^{1} \text { For use with non-bearing trees only. } \\
{ }^{2} \text { For nursery/nonbearing trees only. } \\
{ }^{3} \text { For use with dormant trees only. } \\
{ }^{4} \text { Not registered for use in Miami-Dade, Palm Beach, Broward Counties (see label for others). }\end{array}$} \\
\hline
\end{tabular}


Table 2. Pesticides registered for avocado in Florida.

\begin{tabular}{|c|c|c|c|}
\hline Class & Chemical name & Brand name(s) & Pest(s) controlled \\
\hline \multirow[t]{21}{*}{ Insecticide } & azadirachtin & Align, Azatin & general insecticide \\
\hline & spinosad & Spintor & lepidoptera \\
\hline & pyrethrin + rotenone & Pyrellin & aphids, lepidoptera, thrips \\
\hline & sulfur & Sulfur 6L, Sulfur Flowable, Thiolux & mites \\
\hline & fenoxycarb & Logic $^{2}$, Precision $^{2}$ & ants \\
\hline & malathion & Malathion & thrips, scales, lepidoptera \\
\hline & permethrin & Pounce, Ambush & $\begin{array}{l}\text { thrips, lepidoptera, lace } \\
\text { bug, mirids, Brown soft } \\
\text { scale, leaf hopper }\end{array}$ \\
\hline & various refined horticultural oils & $\begin{array}{l}\text { Sunspray, citrus spray oil, crop } \\
\text { oil, FC } 435-66 \text {, FC } 455-88 \text {, others }\end{array}$ & $\begin{array}{l}\text { various aphids, mites, } \\
\text { scales }\end{array}$ \\
\hline & potassium salts of fatty acids & Safer Soap & $\begin{array}{l}\text { aphids, lace bug, plant } \\
\text { bugs, mites, thrips, scales }\end{array}$ \\
\hline & methomyl & Lannate & lepidoptera \\
\hline & Bacillus thuringiensis & Dipel, others & lepidoptera \\
\hline & Beauveria bassiana & Mycotrol & lace bug \\
\hline & pymetrozine & Endeavor $^{2}$ & whiteflies, aphids, \\
\hline & kaolin (clay) & Surround & $\begin{array}{l}\text { barrier and irritant to } \\
\text { various insects }\end{array}$ \\
\hline & S-methoprene & Extinguish & ants \\
\hline & hydramethylnon & Amdro $^{1}$ & ants \\
\hline & pyriproxyfen & Knack, Distance $^{2}$ & scales, thrips, ants \\
\hline & bifenazate & Floramite $^{1}$ & mites \\
\hline & fenpropathrin & Tame $^{2}$ & various insects, mites \\
\hline & heythiazox & Savey $^{2}$ & various insects, mites \\
\hline & bifenthrin & Talstar $^{1}$ & various insects, mites \\
\hline \multirow[t]{2}{*}{ Mollicides } & metaldehyde & Slug \& Snail Killer & slugs and snails \\
\hline & ferric phosphate & Slug \& Snail Bait & slugs and snails \\
\hline \multirow[t]{7}{*}{ Fungicides } & copper & Kocide, others & $\begin{array}{l}\text { anthracnose, Cercospora } \\
\text { leaf spot }\end{array}$ \\
\hline & sulfur & Sulfur 6L, Sulfur Flowable, Thiolux & powdery mildew \\
\hline & mefenoxam & Subdue Maxx & Phytophthora root rot \\
\hline & azoxystrobin & Abound & $\begin{array}{l}\text { anthracnose, rust, } \\
\text { Cercospora leaf spot, } \\
\text { powdery mildew }\end{array}$ \\
\hline & fosetyl-Al & Aliette & Phytophthora root rot \\
\hline & Trichoderma harzianum & Rootshield $^{2}$ & $\begin{array}{l}\text { root system disease } \\
\text { protectent }\end{array}$ \\
\hline & folpet & Folpet & scab \\
\hline \multirow[t]{3}{*}{ Herbicides } & simazine & Princep & $\begin{array}{l}\text { pre-emergence, annual } \\
\text { broadleaf and grass } \\
\text { weeds }\end{array}$ \\
\hline & fluazifop-butyl & Fusilade $^{1}$ & $\begin{array}{l}\text { post-emergence, annual } \\
\text { and perennial grass weeds }\end{array}$ \\
\hline & sethoxydim & Poast $^{1}$ & $\begin{array}{l}\text { post-emergence, annual } \\
\text { and perennial grass weeds }\end{array}$ \\
\hline
\end{tabular}


Table 2. Pesticides registered for avocado in Florida.

\begin{tabular}{|c|c|c|c|}
\hline Class & Chemical name & Brand name(s) & Pest(s) controlled \\
\hline & norflurazon & Solicam & $\begin{array}{l}\text { pre-emergence, annual } \\
\text { broadleaf and grass } \\
\text { weeds }\end{array}$ \\
\hline & oryzalin & Surflan & $\begin{array}{l}\text { pre-emergence, annual } \\
\text { broadleaf and grass } \\
\text { weeds }\end{array}$ \\
\hline & benefin + oryzalin & $\mathrm{XL} 2 \mathrm{G}^{1}$ & $\begin{array}{l}\text { pre-emergence, annual } \\
\text { broadleaf and grass } \\
\text { weeds }\end{array}$ \\
\hline & isoxaben & Gallery $^{1}$ & $\begin{array}{l}\text { pre-emergence broadleaf } \\
\text { weeds }\end{array}$ \\
\hline & glyphosate (systemic) & Roundup, Glyphosate & $\begin{array}{l}\text { post-emergence annual } \\
\text { and perennial weeds, } \\
\text { woody brush, trees, grass }\end{array}$ \\
\hline & sulfosate & Touchdown & $\begin{array}{l}\text { post-emergence annual } \\
\text { and perennial weeds, } \\
\text { woody brush, trees, grass }\end{array}$ \\
\hline & napropamide & Devrinol & $\begin{array}{l}\text { pre-emergence, annual } \\
\text { broadleaf and grass } \\
\text { weeds }\end{array}$ \\
\hline & oxyfluorfen & Goal $^{3}$ & $\begin{array}{l}\text { pre- and postemergence } \\
\text { of certain broadleaf weeds }\end{array}$ \\
\hline & paraquat & Gramoxone Extra & $\begin{array}{l}\text { post-emergence annual } \\
\text { broadleaf and grass } \\
\text { weeds }\end{array}$ \\
\hline & pelargonic acid & Scythe & $\begin{array}{l}\text { broad leaf and grass } \\
\text { weeds }\end{array}$ \\
\hline & trifluralin & Treflan $^{1}$ & $\begin{array}{l}\text { pre-emergence annual } \\
\text { broadleaf andgrass weeds }\end{array}$ \\
\hline Repellent & capsaicin & Deer Off & $\begin{array}{l}\text { deer, rabbit, mouse } \\
\text { repellent }\end{array}$ \\
\hline \multicolumn{4}{|c|}{$\begin{array}{l}{ }^{1} \text { For use with non-bearing trees only. } \\
{ }^{2} \text { For nursery/nonbearing trees only. } \\
{ }^{3} \text { For use with dormant trees only. } \\
{ }^{4} \text { Not registered for use in Miami-Dade, Palm Beach, Broward Counties (see label for others). }\end{array}$} \\
\hline
\end{tabular}

Table 3. Pesticides registered for banana and plantain in Florida.

\begin{tabular}{|l|l|l|l|}
\hline \hline Class & Chemical name & Brand name(s) & Pest(s) controlled \\
\hline Insecticides & ethoprop & Mocap & nematodes, \\
\hline & Myrothecium verrucaria & DiTera & nematodes \\
\hline & buprofezin & Applaud & $\begin{array}{l}\text { nymph stages of scales, } \\
\text { mealybugs, leafhoppers }\end{array}$ \\
\hline & azadirachtin & Align, Azatin & general insecticide \\
\hline & oxamyl & Vydate & nematodes, \\
\hline & Bacillus thuringiensis & Dipel, others & lepidoptera \\
\hline & Beauveria bassiana & Mycotrol & aphids, mealybugs, others \\
\hline & pyrethrin + rotenone & Pyrellin & aphids, lepidoptera, thrips \\
\hline
\end{tabular}


Table 3. Pesticides registered for banana and plantain in Florida.

\begin{tabular}{|c|c|c|c|}
\hline Class & Chemical name & Brand name(s) & Pest(s) controlled \\
\hline & potassium salts of fatty acids & Safer Soap & $\begin{array}{l}\text { aphids, lace bugs, mealy } \\
\text { bugs, spidermites, others }\end{array}$ \\
\hline & pyrethrins & Pyrenone & $\begin{array}{l}\text { aphids, lepidoptera, mites, } \\
\text { thrips }\end{array}$ \\
\hline & various refined horticultural oils & $\begin{array}{l}\text { Sunspray, citrus spray oil, crop } \\
\text { oil, FC 435-66, FC 455-88, } \\
\text { others }\end{array}$ & $\begin{array}{l}\text { various aphids, mites, } \\
\text { scales }\end{array}$ \\
\hline & pymetrozine & Endeavor $^{2}$ & whiteflies, aphids, \\
\hline & kaolin (clay) & Surround & $\begin{array}{l}\text { barrier and irritant to } \\
\text { various insects }\end{array}$ \\
\hline & S-methoprene & Extinguish & ants \\
\hline & hydramethylnon & Amdro $^{1}$ & ants \\
\hline & pyriproxyfen & Knack, Distance ${ }^{2}$ & scales, thrips, ants \\
\hline & bifenazate & Floramite $^{1}$ & mites \\
\hline & fenpropathrin & Tame $^{2}$ & various insects, mites \\
\hline & heythiazox & Savey $^{2}$ & various insects, mites \\
\hline & bifenthrin & Talstar $^{1}$ & various insects, mites \\
\hline Mollicide & metaldehyde & Slug \& Snail Killer & slugs and snails \\
\hline \multirow[t]{13}{*}{ Fungicides } & mancozeb & Manzate, Dithane & yellow and black Sigatoka \\
\hline & copper & Kocide, others & $\begin{array}{l}\text { anthracnose, Sigatoka, } \\
\text { other various diseases }\end{array}$ \\
\hline & azoxystrobin & Abound & yellow and black Sigatoka \\
\hline & fenbuconazole & Enable & yellow and black Sigatoka \\
\hline & fosetyl-Al & Aliette & yellow and black Sigatoka \\
\hline & maneb & Manex & yellow and black Sigatoka \\
\hline & tebuconazole & Elite & yellow and black Sigatoka \\
\hline & thiabendaxole (TBZ) & Decoo Salt No. 19 (banana only) & postharvest fungicide \\
\hline & sulfur & Sulfur 6L, Sulfur Flowable, Thiolux & $\begin{array}{l}\text { various diseases (some } \\
\text { mites) }\end{array}$ \\
\hline & mefenoxam & Subdue Maxx ${ }^{2}$ & root rot \\
\hline & Trichoderma harzianum & Rootshield $^{2}$ & $\begin{array}{l}\text { root system disease } \\
\text { protectent }\end{array}$ \\
\hline & carbonic acid & Kaligreen & general fungicide \\
\hline & hydrogen peroxide & Oxidate & biocide \\
\hline \multirow[t]{5}{*}{ Herbicides } & ametryn & Evik & $\begin{array}{l}\text { annual broadleaf and grass } \\
\text { weeds }\end{array}$ \\
\hline & diruon & Karmex & $\begin{array}{l}\text { annual broadleaf and grass } \\
\text { weeds }\end{array}$ \\
\hline & glyphosate (systemic) (systemic) & Roundup, Glyphosate & broad leaf and grass weeds \\
\hline & paraquat (contact) & Gramoxone Extra & broad leaf and grass weeds \\
\hline & sulfosate (systemic) & Touchdown & broad leaf and grass weeds \\
\hline
\end{tabular}


Table 3. Pesticides registered for banana and plantain in Florida.

\begin{tabular}{|c|c|c|c|}
\hline Class & Chemical name & Brand name(s) & Pest(s) controlled \\
\hline & pelargonic acid & Scythe & $\begin{array}{l}\text { broad leaf and grass } \\
\text { weeds }\end{array}$ \\
\hline Repellent & capsaicin & Deer Off & $\begin{array}{l}\text { deer, rabbit, mouse } \\
\text { repellent }\end{array}$ \\
\hline \multicolumn{4}{|c|}{$\begin{array}{l}{ }^{1} \text { For use with non-bearing trees only. } \\
{ }^{2} \text { For nursery/nonbearing trees only. } \\
{ }^{3} \text { For use with dormant trees only. } \\
{ }^{4} \text { Not registered for use in Miami-Dade, Palm Beach, Broward Counties (see label for others). }\end{array}$} \\
\hline
\end{tabular}

Table 4. Pesticides registered for Barbados cherry in Florida.

\begin{tabular}{|c|c|c|c|}
\hline Class & Chemical name & Brand name(s) & Pest(s) controlled \\
\hline \multirow[t]{18}{*}{ Insectides } & pyrethrin + rotenone & Pyrellin & aphids, lepidoptera, thrips \\
\hline & potassium salts of fatty acids & Safer Soap & $\begin{array}{l}\text { aphids, lace bugs, mealy } \\
\text { bugs, spidermites, others }\end{array}$ \\
\hline & Beauveria bassiana & Mycotrol & aphids, mealybugs, others \\
\hline & fenoxycarb & Precision & ants \\
\hline & spinosad & Spintor & lepidoptera larvae \\
\hline & azadirachtin & Align, Azatin & general insecticide \\
\hline & various refined horticultural oils & $\begin{array}{l}\text { Sunspray, citrus spray oil, crop } \\
\text { oil, FC 435-66, FC 455-88, others }\end{array}$ & $\begin{array}{l}\text { various aphids, mites, } \\
\text { scales }\end{array}$ \\
\hline & fluazifop-p-butyl & Fusilade & $\begin{array}{l}\text { post-emergence, annual } \\
\text { and perennial grass weeds }\end{array}$ \\
\hline & pyrethrins & Pyrenone & $\begin{array}{l}\text { aphids, lepidoptera, mites, } \\
\text { thrips }\end{array}$ \\
\hline & pymetrozine & Endeavor $^{2}$ & whiteflies, aphids, \\
\hline & kaolin (clay) & Surround & $\begin{array}{l}\text { barrier and irritant to } \\
\text { various insects }\end{array}$ \\
\hline & S-methoprene & Extinguish & ants \\
\hline & hydramethylnon & Amdro $^{1}$ & ants \\
\hline & pyriproxyfen & Knack, Distance $^{2}$ & scales, thrips, ants \\
\hline & bifenazate & Floramite $^{1}$ & mites \\
\hline & fenpropathrin & Tame $^{2}$ & various insects, mites \\
\hline & heythiazox & Savey $^{2}$ & various insects, mites \\
\hline & bifenthrin & Talstar $^{1}$ & various insects, mites \\
\hline \multirow[t]{3}{*}{ Fungicide } & azoxystrobin & Abound & anthracnose \\
\hline & mefenoxam & Subdue Maxx ${ }^{2}$ & root rot \\
\hline & Trichoderma harzianum & Rootshield $^{2}$ & $\begin{array}{l}\text { root system disease } \\
\text { protectent }\end{array}$ \\
\hline \multirow[t]{4}{*}{ Herbicides } & paraquat & Gramoxone Extra & broad leaf and grass weeds \\
\hline & glyphosate (systemic) & Roundup, Glyphosate & broad leaf and grass weeds \\
\hline & fluazifop-butyl & Fusilade $^{1}$ & $\begin{array}{l}\text { post-emergence, annual } \\
\text { and perennial grass weeds }\end{array}$ \\
\hline & pelargonic acid & Scythe & broad leaf and grass weeds \\
\hline
\end{tabular}


Table 4. Pesticides registered for Barbados cherry in Florida.

\begin{tabular}{|c|c|c|c|}
\hline Class & Chemical name & Brand name(s) & Pest(s) controlled \\
\hline & napropamide & Devrinol & $\begin{array}{l}\text { broad leaf and grass } \\
\text { weeds }\end{array}$ \\
\hline \multicolumn{4}{|c|}{$\begin{array}{l}{ }^{1} \text { For use with non-bearing trees only. } \\
2 \text { For nursery/nonbearing trees only. } \\
3 \text { For use with dormant trees only. } \\
{ }^{3} \text { Not registered for use in Miami-Dade, Plam Beach, Broward Counties (see label for others). }\end{array}$} \\
\hline
\end{tabular}

Table 5. Pesticides registered for black sapote in Florida.

\begin{tabular}{|c|c|c|c|}
\hline Class & Chemical name & Brand name(s) & Pest(s) controlled \\
\hline \multirow[t]{10}{*}{ Insecticides } & pyrethrin + rotenone & Pyrellin & aphids, lepidoptera, thrips \\
\hline & potassium salts of fatty acids & Safer Soap & $\begin{array}{l}\text { aphids, lace bugs, mealy } \\
\text { bugs, spidermites, others }\end{array}$ \\
\hline & various refined horticultural oils & $\begin{array}{l}\text { Sunspray, citrus spray oil, crop } \\
\text { oil, FC } 435-66 \text {, FC } 455-88 \text {, others }\end{array}$ & $\begin{array}{l}\text { various aphids, mites, } \\
\text { scales }\end{array}$ \\
\hline & spinosad & Spintor & lepidoptera larvae \\
\hline & azadirachtin & Align, Azatin & general insecticide \\
\hline & Beauveria bassiana & Mycotrol & aphids, mealybugs, others \\
\hline & Bacillus thuringiensis & Dipel, others & lepidoptera larvae \\
\hline & pymetrozine & Endeavor $^{2}$ & whiteflies, aphids, \\
\hline & pyriproxyfen & Knack, Distance ${ }^{2}$ & \\
\hline & kaolin (clay) & Surround & $\begin{array}{l}\text { barrier and irritant to } \\
\text { various insects }\end{array}$ \\
\hline \multirow[t]{3}{*}{ Fungicides } & mefenoxam & Subdue Maxx ${ }^{2}$ & root rot \\
\hline & azoxystrobin & Abound & anthracnose \\
\hline & Trichoderma harzianum & Rootshield $^{2}$ & $\begin{array}{l}\text { root system disease } \\
\text { protectent }\end{array}$ \\
\hline \multirow[t]{2}{*}{ Herbicide } & glyphosate (systemic) & Roundup, Glyphosate & broad leaf and grass weeds \\
\hline & pelargonic acid & Scythe & broad leaf and grass weeds \\
\hline Repellent & capsaicin & Deer Off & $\begin{array}{l}\text { deer, rabbit, mouse } \\
\text { repellent }\end{array}$ \\
\hline \multicolumn{4}{|c|}{$\begin{array}{l}{ }^{1} \text { For use with non-bearing trees only. } \\
{ }^{2} \text { For nursery/nonbearing trees only. } \\
{ }^{3} \text { For use with dormant trees only. } \\
{ }^{4} \text { Not registered for use in Miami-Dade, Palm Beach, Broward Counties (see label for others). }\end{array}$} \\
\hline
\end{tabular}

Table 6. Pesticides registered for carambola in Florida.

\begin{tabular}{|l|l|l|l|}
\hline \hline Class & Chemical name & Brand name(s) & Pest(s) controlled \\
\hline Insecticides & pyrethrin + rotenone & Pyrellin & aphids, lepidoptera, thrips \\
\hline & potassium salts of fatty acids & Safer Soap & $\begin{array}{l}\text { aphids, lace bugs, mealy } \\
\text { bugs, spidermites, others }\end{array}$ \\
\hline & various refined horticultural oils & $\begin{array}{l}\text { Sunspray, citrus spray oil, crop } \\
\text { oil, FC 435-66, FC 455-88, others }\end{array}$ & various aphids, mites, scales \\
& spinosad & Spintor & lepidoptera larvae \\
\hline \hline
\end{tabular}


Table 6. Pesticides registered for carambola in Florida.

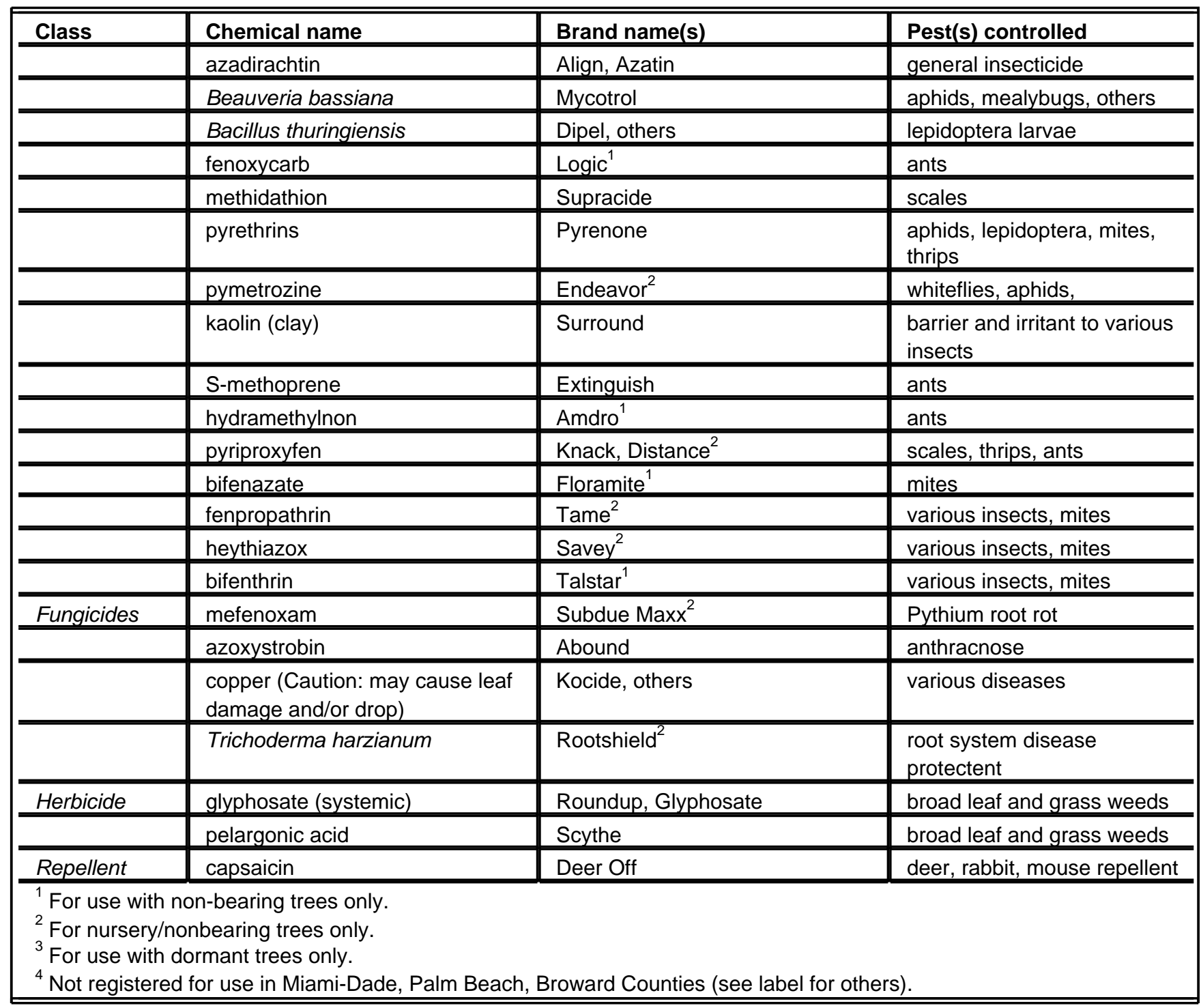

Table 7. Pesticides registered for ccoconut in Florida.

\begin{tabular}{|l|l|l|l|}
\hline \hline Class & Chemical name & Brand name(s) & Pest(s) controlled \\
\hline Insecticides & various refined horticultural oils & $\begin{array}{l}\text { Sunspray, citrus spray oil, crop } \\
\text { oil, FC 435-66, FC 455-88, } \\
\text { others }\end{array}$ & various aphids, mites, scales \\
\hline & Bacillus thuringiensis & Dipel, others & lepidoptera larvae \\
\hline & Beauveria bassiana & Mycotrol & aphids, mealybugs, others \\
\hline & potassium salts of fatty acids & Safer Soap & $\begin{array}{l}\text { aphids, lace bugs, mealy } \\
\text { bugs, spidermites, others }\end{array}$ \\
\hline & azadirachtin & general insecticide \\
\hline & pymetrozine & Align, Azatin & whiteflies, aphids, \\
\hline & kaolin (clay) & Endeavor ${ }^{2}$ & $\begin{array}{l}\text { barrier and irritant to various } \\
\text { insects }\end{array}$ \\
\hline Fungicide & mefenoxam & Surround & root rot \\
\hline \hline
\end{tabular}


Table 7. Pesticides registered for ccoconut in Florida.

\begin{tabular}{|c|c|c|c|}
\hline Class & Chemical name & Brand name(s) & Pest(s) controlled \\
\hline & Trichoderma harzianum & Rootshield $^{2}$ & $\begin{array}{l}\text { root system disease } \\
\text { protectent }\end{array}$ \\
\hline \multirow[t]{3}{*}{ Herbicide } & glyphosate (systemic) & Roundup, Glyphosate & broad leaf and grass weeds \\
\hline & pelargonic acid & Scythe & broad leaf and grass weeds \\
\hline & pelargonic acid & Scythe & broad leaf and grass weeds \\
\hline Repellent & capsaicin & Deer Off & deer, rabbit, mouse repellent \\
\hline \multicolumn{4}{|c|}{$\begin{array}{l}{ }^{1} \text { For use with non-bearing trees only. } \\
{ }^{2} \text { For nursery/nonbearing trees only. } \\
{ }^{3} \text { For use with dormant trees only. }\end{array}$} \\
\hline
\end{tabular}

Table 8. Pesticides registered for guava in Florida.

\begin{tabular}{|c|c|c|c|}
\hline Class & Chemical name & Brand name(s) & Pest(s) controlled \\
\hline \multirow[t]{18}{*}{ Insecticides } & pyrethrin + rotenone & Pyrellin & aphids, lepidoptera, thrips \\
\hline & potassium salts of fatty acids & Safer Soap & $\begin{array}{l}\text { aphids, lace bugs, mealy } \\
\text { bugs, spidermites, others }\end{array}$ \\
\hline & various refined horticultural oils & $\begin{array}{l}\text { Sunspray, citrus spray oil, crop } \\
\text { oil, FC 435-66, FC 455-88, } \\
\text { others }\end{array}$ & various aphids, mites, scales \\
\hline & spinosad & Spintor & lepidoptera larvae \\
\hline & azadirachtin & Align, Azatin & general insecticide \\
\hline & Beauveria bassiana & Mycotrol & aphids, mealybugs, others \\
\hline & malathion & Malathion & $\begin{array}{l}\text { thrips, scales, lepidoptera, } \\
\text { Caribbean fruit fly }\end{array}$ \\
\hline & fenoxycarb & Logic $^{2}$ & ants \\
\hline & Bacillus thuringiensis & Dipel, others & lepidoptera larvae \\
\hline & pymetrozine & Endeavor $^{2}$ & whiteflies, aphids, \\
\hline & kaolin (clay) & Surround & $\begin{array}{l}\text { barrier and irritant to various } \\
\text { insects }\end{array}$ \\
\hline & S-methoprene & Extinguish & ants \\
\hline & hydramethylnon & Amdro $^{1}$ & ants \\
\hline & pyriproxyfen & Knack, Distance ${ }^{2}$ & scales, thrips, ants \\
\hline & bifenazate & Floramite $^{1}$ & mites \\
\hline & fenpropathrin & Tame $^{2}$ & various insects, mites \\
\hline & heythiazox & Savey $^{2}$ & various insects, mites \\
\hline & bifenthrin & Talstar $^{1}$ & various insects, mites \\
\hline \multirow[t]{4}{*}{ Fungicides } & azoxystrobin & Abound & anthracnose \\
\hline & copper & Kocide, others & $\begin{array}{l}\text { anthracnose, various other } \\
\text { diseases }\end{array}$ \\
\hline & mefenoxam & Subdue Maxx ${ }^{2}$ & root rot \\
\hline & Trichoderma harzianum & Rootshield $^{2}$ & root system disease protectent \\
\hline \multirow[t]{4}{*}{ Herbicide } & glyphosate (systemic) & Roundup, Glyphosate & broad leaf and grass weeds \\
\hline & pelargonic acid & Scythe & broad leaf and grass weeds \\
\hline & oxyfluorfen & Goal & $\begin{array}{l}\text { pre-emergence on broadleaf } \\
\text { weeds }\end{array}$ \\
\hline & paraquat & Gramoxone Extra & broad leaf and grass weeds \\
\hline
\end{tabular}


Table 8. Pesticides registered for guava in Florida.

\begin{tabular}{l|l|l|l|}
\hline \hline Class & Chemical name & Brand name(s) & Pest(s) controlled \\
\hline & fluazifop-butyl & Fusilade ${ }^{1}$ & $\begin{array}{l}\text { post-emergence, annual and } \\
\text { perennial grass weeds }\end{array}$ \\
\hline & oryzalin & Surflan & $\begin{array}{l}\text { annual grasses and certain } \\
\text { broadleaf weeds }\end{array}$ \\
\hline & pelargonic acid & Scythe & broad leaf and grass weeds \\
\hline Repellent & capsaicin & Deer Off & deer, rabbit, mouse repellent \\
\hline $\begin{array}{l}{ }^{2} \text { For use with non-bearing trees only. } \\
{ }^{3} \text { For nursery/nonbearing trees only. }\end{array}$ & \\
${ }^{4}$ Not registered for use in Miami-Dade, Palm Beach, Broward Counties (see label for others). \\
\hline \hline
\end{tabular}

Table 9. Pesticides registered for jackfruit in Florida.

\begin{tabular}{|c|c|c|c|}
\hline Class & Chemical name & Brand name(s) & Pest(s) controlled \\
\hline \multirow[t]{16}{*}{ Insecticides } & potassium salts of fatty acids & Safer Soap & $\begin{array}{l}\text { aphids, lace bugs, mealy bugs, } \\
\text { spidermites, others }\end{array}$ \\
\hline & various refined horticultural oils & $\begin{array}{l}\text { Sunspray, citrus spray oil, crop } \\
\text { oil, FC 435-66, FC 455-88, } \\
\text { others }\end{array}$ & various aphids, mites, scales \\
\hline & spinosad & Spintor & lepidoptera larvae \\
\hline & azadirachtin & Align, Azatin & general insecticide \\
\hline & pyrethrins & Pyronyl & aphids, thrips \\
\hline & Bacillus thuringiensis & Dipel, others & lepidoptera larvae \\
\hline & Beauveria bassiana & Mycotrol & aphids, mealybugs, others \\
\hline & pymetrozine & Endeavor $^{2}$ & whiteflies, aphids, \\
\hline & kaolin (clay) & Surround & $\begin{array}{l}\text { barrier and irritant to various } \\
\text { insects }\end{array}$ \\
\hline & S-methoprene & Extinguish & ants \\
\hline & hydramethylnon & Amdro $^{1}$ & ants \\
\hline & pyriproxyfen & Knack, Distance ${ }^{2}$ & scales, thrips, ants \\
\hline & bifenazate & Floramite $^{1}$ & mites \\
\hline & fenpropathrin & Tame $^{2}$ & various insects, mites \\
\hline & heythiazox & Savey $^{2}$ & various insects, mites \\
\hline & bifenthrin & Talstar $^{1}$ & various insects, mites \\
\hline \multirow[t]{3}{*}{ Fungicides } & azoxystrobin & Abound & anthracnose \\
\hline & mefenoxam & Subdue Maxx ${ }^{2}$ & root rot \\
\hline & Trichoderma harzianum & Rootshield $^{2}$ & root system disease protectent \\
\hline \multirow[t]{2}{*}{ Herbicide } & pelargonic acid & Scythe & broad leaf and grass weeds \\
\hline & glyphosate (systemic) & Roundup, Glyphosate & broad leaf and grass weeds \\
\hline Repellent & capsaicin & Deer Off & deer, rabbit, mouse repellent \\
\hline $\begin{array}{l}{ }^{1} \text { For use witt } \\
{ }^{2} \text { For nursery } \\
{ }^{3} \text { For use witr } \\
{ }^{4} \text { Not register }\end{array}$ & $\begin{array}{l}\text { n-bearing trees only. } \\
\text { nbearing trees only. } \\
\text { ormant trees only. } \\
\text { for use in Miami-Dade, }\end{array}$ & 0 & \\
\hline
\end{tabular}


Table 10. Pesticides registered for 'Tahiti' lime, Key lime, kumquat, and pummelo in Florida.

\begin{tabular}{|c|c|c|c|}
\hline Class & Chemical name & Brand name(s) & Pest(s) controlled \\
\hline \multirow[t]{35}{*}{ Insecticides } & various refined horticultural oils & $\begin{array}{l}\text { Sunspray, citrus spray oil, crop } \\
\text { oil, FC 435-66, FC 455-88, } \\
\text { others }\end{array}$ & various aphids, mites, scales \\
\hline & sulfur & $\begin{array}{l}\text { Sulfur } 6 \mathrm{~L}, \text { Sulfur Flowable, } \\
\text { Thiolux }\end{array}$ & various mites \\
\hline & fenoxycarb & $\operatorname{Logic}^{1}$ & ants \\
\hline & abamectin & Agri-Mek & mites \\
\hline & formetanate & Carzol SP (limes only) & thrips \\
\hline & buprofezin & Applaud & scales, mealybug, leafhoppers \\
\hline & cryolite & Kyrocide & lepidoptera \\
\hline & imidacloprid & Provado & $\begin{array}{l}\text { aphids, leafminers, scales, } \\
\text { leafhoppers, mealybugs }\end{array}$ \\
\hline & oxamyl & Vydate & $\begin{array}{l}\text { rust mites, thrips, nematode } \\
\text { suppression }\end{array}$ \\
\hline & oxydemeton-methyl & Metasystox ${ }^{1}$ & mites, leafminers \\
\hline & propargite & Omite $^{1}$ & mites \\
\hline & fenpropathrin & Danitol & $\begin{array}{l}\text { thrips, mites, sharpshooters, } \\
\text { adult weevils }\end{array}$ \\
\hline & kaolin (clay) & Surround & $\begin{array}{l}\text { barrier and irritant to various } \\
\text { insects }\end{array}$ \\
\hline & dicofol & Kelthane & mites \\
\hline & ethion & Ethion (limes only) & mites, scales \\
\hline & diflubenzuron & Micromite & $\begin{array}{l}\text { leafminers, weevil eggs/larvae, } \\
\text { rust mite }\end{array}$ \\
\hline & fenbutatin-oxide & Vendex, Hexakis & miticide \\
\hline & dimethoate & Dimethoate, Cygon & miticide \\
\hline & Beauveria bassiana & Mycotrol & aphids, mealybugs, others \\
\hline & Bacillus thuringiensis & Dipel, others & lepidoptera larvae \\
\hline & chlorpyrifos & Lorsban & $\begin{array}{l}\text { aphids, lepidoptera, scales, } \\
\text { thrips }\end{array}$ \\
\hline & malathion & Malthion & $\begin{array}{l}\text { thrips, scales, lepidoptera, } \\
\text { Caribbean fruit fly }\end{array}$ \\
\hline & pyrethrin + rotenone & Pyrellin & aphids, lepidoptera, thrips \\
\hline & potassium salts of fatty acids & Safer Soap & $\begin{array}{l}\text { aphids, lace bugs, mealy bugs, } \\
\text { spidermites, others }\end{array}$ \\
\hline & methidathion & Supracide & scales \\
\hline & acephate & Acephate $^{2}$ & $\begin{array}{l}\text { aphids, plant bugs, } \\
\text { grasshoppers, thrips, ants }\end{array}$ \\
\hline & azadirachtin & Align, Azatin & general insecticide \\
\hline & carbaryl & Sevin & $\begin{array}{l}\text { citrus root weevil adults, mites, } \\
\text { lepidoptera }\end{array}$ \\
\hline & fenamiphos & Nemacur & nematodes \\
\hline & pyridabin & Nexter & mites \\
\hline & pymetrozine & Endeavor $^{2}$ & whiteflies, aphids, \\
\hline & S-methoprene & Extinguish & ants \\
\hline & hydramethylnon & Amdro $^{1}$ & ants \\
\hline & pyriproxyfen & Knack, Distance $^{2}$ & scales, thrips, ants \\
\hline & bifenazate & Floramite $^{1}$ & mites \\
\hline
\end{tabular}


Table 10. Pesticides registered for 'Tahiti' lime, Key lime, kumquat, and pummelo in Florida.

\begin{tabular}{|c|c|c|c|}
\hline Class & Chemical name & Brand name(s) & Pest(s) controlled \\
\hline & fenpropathrin & Tame $^{2}$ & various insects, mites \\
\hline & heythiazox & Savey $^{2}$ & various insects, mites \\
\hline & bifenthrin & Talstar $^{1}$ & various insects, mites \\
\hline \multirow[t]{7}{*}{ Fungicides } & copper & Kocide, others & Greasy spot, melanose \\
\hline & azoxystrobin & Abound & anthracnose \\
\hline & fosetyl-Al & Aliette & phytophthora root rot \\
\hline & mefenoxam & Subdue Maxx & phytophthora root rot \\
\hline & ferbam & Ferbam Granuflo (limes only) & $\begin{array}{l}\text { post bloom fruit drop, scab, } \\
\text { anthracnose }\end{array}$ \\
\hline & Trichoderma harzianum & Rootshield $^{2}$ & root system disease protectent \\
\hline & hydrogen peroxide & & biocide \\
\hline \multirow[t]{11}{*}{ Herbicides } & bromacil & Hyvar $X^{4}$ & grass weeds \\
\hline & diuron & Karmex & $\begin{array}{l}\text { annual broadleaf and grass } \\
\text { weeds }\end{array}$ \\
\hline & bromacil + diuron & Krovar & $\begin{array}{l}\text { annual broadleaf and grass } \\
\text { weeds }\end{array}$ \\
\hline & norflurazon & Solicam & grass weeds \\
\hline & oryzalin & Surflan & $\begin{array}{l}\text { annual grasses and certain } \\
\text { broadleaf weeds }\end{array}$ \\
\hline & glyphosate (systemic) & Roundup, Glyphosate & broad leaf and grass weeds \\
\hline & paraquat & Gramoxone Extra & broad leaf and grass weeds \\
\hline & fluazifop-p-butyl & Fusilade $^{1}$ & $\begin{array}{l}\text { post-emergence, annual and } \\
\text { perennial grass weeds }\end{array}$ \\
\hline & sethoxydim & Poast & grass weeds \\
\hline & Phytophthora palmivora & Devine & specific vines \\
\hline & pelargonic acid & Scythe & broad leaf and grass weeds \\
\hline Repellent & capsaicin & Deer Off & deer, rabbit, mouse repellent \\
\hline \multicolumn{4}{|c|}{$\begin{array}{l}{ }^{1} \text { For use with non-bearing trees only. } \\
{ }^{2} \text { For nursery/nonbearing trees only. } \\
{ }^{3} \text { For use with dormant trees only. } \\
{ }^{4} \text { Not registered for use in Miami-Dade, Palm Beach, Broward Counties (see label for others). }\end{array}$} \\
\hline
\end{tabular}

Table 11. Pesticides registered for lychee and longan in Florida.

\begin{tabular}{|l|l|l|l|}
\hline \hline Class & Chemical name & Brand name(s) & Pest(s) controlled \\
\hline Insecticides & methidathion & Supracide (longan only) & scales \\
\hline & pyrethrin + rotenone & Pyrellin & aphids, lepidoptera, thrips \\
\hline & potassium salts of fatty acids & Safer Soap & $\begin{array}{l}\text { aphids, lace bugs, mealy bugs, } \\
\text { spidermites, others }\end{array}$ \\
\hline & methoxyfenozide & Intrepid & lychee web worm \\
\hline & spinosad & Spintor & lepidoptera larvae \\
\hline & azadirachtin & Align, Azatin & general insecticide \\
\hline & Beauveria bassiana & Mycotrol & aphids, mealybugs, others \\
\hline & various refined horticultural oils & Sunspray, citrus spray oil, crop & various aphids, mites, scales \\
& & $\begin{array}{l}\text { oil, FC 435-66, FC 455-88, } \\
\text { others }\end{array}$ & ants \\
\hline \hline
\end{tabular}


Table 11. Pesticides registered for lychee and longan in Florida.

\begin{tabular}{|c|c|c|c|}
\hline \multirow[t]{12}{*}{ Class } & Chemical name & Brand name(s) & Pest(s) controlled \\
\hline & kaolin (clay) & Surround & $\begin{array}{l}\text { barrier and irritant to various } \\
\text { insects }\end{array}$ \\
\hline & Bacillus thuringiensis & Dipel, others & lepidoptera larvae \\
\hline & pymetrozine & Endeavor $^{2}$ & whiteflies, aphids, \\
\hline & S-methoprene & Extinguish & ants \\
\hline & hydramethylnon & Amdro $^{1}$ & ants \\
\hline & pyriproxyfen & Knack & scales, thrips, ants \\
\hline & bifenazate & Floramite $^{1}$ & mites \\
\hline & fenpropathrin & Tame $^{2}$ & various insects, mites \\
\hline & heythiazox & Savey $^{2}$ & various insects, mites \\
\hline & bifenthrin & Talstar $^{1}$ & various insects, mites \\
\hline & pyriproxyfen & Knack, Distance $^{2}$ & \\
\hline \multirow[t]{4}{*}{ Fungicides } & copper (lychee only) & Kocide, others & anthracnose \\
\hline & azoxystrobin & Abound & anthracnose \\
\hline & Trichoderma harzianum & Rootshield & root system disease protectent \\
\hline & mefenoxam & Subdue Maxx ${ }^{2}$ & root rot \\
\hline \multirow[t]{2}{*}{ Herbicide } & glyphosate (systemic) & Roundup, Glyphosate & broad leaf and grass weeds \\
\hline & pelargonic acid & Scythe & broad leaf and grass weeds \\
\hline Repellent & capsaicin & Deer Off & deer, rabbit, mouse repellent \\
\hline \multicolumn{4}{|c|}{$\begin{array}{l}{ }^{1} \text { For use with non-bearing trees only. } \\
{ }^{2} \text { For nursery/nonbearing trees only. } \\
{ }^{3} \text { For use with dormant trees only. } \\
{ }^{4} \text { Not registered for use in Miami-Dade }\end{array}$} \\
\hline
\end{tabular}

Table 12. Pesticides registered for mamey sapote in Florida.

\begin{tabular}{|c|c|c|c|}
\hline Class & Chemical name & Brand name(s) & Pest(s) controlled \\
\hline \multirow[t]{15}{*}{ Insecticides } & pyrethrin + rotenone & Pyrellin & aphids, lepidoptera, thrips \\
\hline & potassium salts of fatty acids & Safer Soap & $\begin{array}{l}\text { aphids, lace bugs, mealy bugs, } \\
\text { spidermites, others }\end{array}$ \\
\hline & spinosad & Spintor & lepidoptera larvae \\
\hline & azadirachtin & Align, Azatin & general insecticide \\
\hline & Beauveria bassiana & Mycotrol & aphids, mealybugs, thrips, others \\
\hline & various refined horticultural oils & $\begin{array}{l}\text { Sunspray, citrus spray oil, crop } \\
\text { oil, FC 435-66, FC 455-88, } \\
\text { others }\end{array}$ & various aphids, mites, scales \\
\hline & fenoxycarb & $\operatorname{Logic}^{1}$ & ants \\
\hline & kaolin clay & Surround & $\begin{array}{l}\text { barrier and irritant to various } \\
\text { insects }\end{array}$ \\
\hline & Bacillus thuringiensis & Dipel, others & lepidoptera larvae \\
\hline & pymetrozine & Endeavor $^{2}$ & whiteflies, aphids, \\
\hline & S-methoprene & Extinguish & ants \\
\hline & hydramethylnon & Amdro $^{1}$ & ants \\
\hline & pyriproxyfen & Knack, Distance $^{2}$ & scales, thrips, ants \\
\hline & bifenazate & Floramite $^{1}$ & mites \\
\hline & fenpropathrin & Tame $^{2}$ & various insects, mites \\
\hline
\end{tabular}


Table 12. Pesticides registered for mamey sapote in Florida.

\begin{tabular}{|c|c|c|c|}
\hline Class & Chemical name & Brand name(s) & Pest(s) controlled \\
\hline & heythiazox & Savey $^{2}$ & various insects, mites \\
\hline & bifenthrin & Talstar $^{1}$ & various insects, mites \\
\hline \multirow[t]{4}{*}{ Fungicides } & copper & Kocide, others & red alga leaf spot \\
\hline & azoxystrobin & Abound & anthracnose \\
\hline & mefenoxam & Subdue Maxx & Pythium root rot \\
\hline & Trichoderma harzianum & Rootshield $^{2}$ & root system disease protectent \\
\hline \multirow[t]{2}{*}{ Herbicide } & glyphosate (systemic) & Roundup, Glyphosate & broad leaf and grass weeds \\
\hline & pelargonic acid & Scythe & broad leaf and grass weeds \\
\hline Repellent & capsaicin & Deer Off & deer, rabbit, mouse repellent \\
\hline \multicolumn{4}{|c|}{$\begin{array}{l}{ }^{1} \text { For use with non-bearing trees only. } \\
2 \text { For nursery/nonbearing trees only. } \\
{ }^{3} \text { For use with dormant trees only. } \\
{ }^{4} \text { Not registered for use in Miami-Dade, Palm Beach, Broward Counties (see label for others). }\end{array}$} \\
\hline
\end{tabular}

Table 13. Pesticides registered for mango in Florida.

\begin{tabular}{|c|c|c|c|}
\hline Class & Chemical name & Brand name(s) & Pest(s) controlled \\
\hline \multirow[t]{23}{*}{ Insecticides } & pyrethrin + rotenone & Pyrellin & aphids, lepidoptera, thrips \\
\hline & potassium salts of fatty acids & Safer Soap & $\begin{array}{l}\text { aphids, lace bugs, mealy bugs, } \\
\text { spidermites, others }\end{array}$ \\
\hline & spinosad & Spintor & lepidoptera larvae \\
\hline & azadirachtin & Align, Azatin & general insecticide \\
\hline & Beauveria bassiana & Mycotrol & aphids, mealybugs, thrips, others \\
\hline & Bacillus thuringiensis & Dipel, others & lepidoptera larvae \\
\hline & various refined horticultural oils & $\begin{array}{l}\text { Sunspray, citrus spray oil, } \\
\text { crop oil, FC } 435-66, \text { FC } \\
455-88 \text {, others }\end{array}$ & various aphids, mites, scales \\
\hline & fenoxycarb & $\operatorname{Logic}^{1}$ & ants \\
\hline & pyrethrins & Pyrenone & aphids, lepidoptera, mites, thrips \\
\hline & malathion & Malthion & thrips, scales \\
\hline & imidacloprid & Provado & thrips \\
\hline & methidathion & Supracide & scales \\
\hline & sulfur & $\begin{array}{l}\text { Sulfur 6L, Sulfur Flowable, } \\
\text { Thiolux }\end{array}$ & mites \\
\hline & Myrothecium verruearia & DiTera & nematodes \\
\hline & harpin & Messenger & $\begin{array}{l}\text { general plant disease and insect } \\
\text { management }\end{array}$ \\
\hline & pymetrozine & Endeavor $^{2}$ & whiteflies, aphids, \\
\hline & kaolin (clay) & Surround & $\begin{array}{l}\text { barrier and irritant to various } \\
\text { insects }\end{array}$ \\
\hline & S-methoprene & Extinguish & ants \\
\hline & hydramethylnon & Amdro $^{1}$ & ants \\
\hline & pyriproxyfen & Knack & scales, thrips, ants \\
\hline & bifenazate & Floramite $^{1}$ & mites \\
\hline & fenpropathrin & Tame $^{2}$ & various insects, mites \\
\hline & heythiazox & Savey $^{2}$ & various insects, mites \\
\hline
\end{tabular}


Table 13. Pesticides registered for mango in Florida.

\begin{tabular}{|c|c|c|c|}
\hline Class & Chemical name & Brand name(s) & Pest(s) controlled \\
\hline & bifenthrin & Talstar $^{1}$ & various insects, mites \\
\hline & pyriproxyfen & Knack, Distance ${ }^{2}$ & \\
\hline \multirow[t]{8}{*}{ Fungicides } & sulfur & $\begin{array}{l}\text { Sulfur 6L, Sulfur Flowable, } \\
\text { Thiolux }\end{array}$ & powdery mildew \\
\hline & copper & Kocide, others & anthracnose, red alga \\
\hline & azoxystrobin & Abound & anthracnose \\
\hline & mefenoxam & Subdue Maxx & Pythium root rot \\
\hline & ferbam & Ferbam Granuflo & anthracnose \\
\hline & potassium bicarbonate & Armicarb 100 & powdery mildew \\
\hline & Trichoderma harzianum & Rootshield $^{2}$ & root system disease protectent \\
\hline & thiabendaxole (TBZ) & Mertect, Decoo Salt No. 19 & postharvest fungicide \\
\hline \multirow[t]{3}{*}{ Herbicide } & glyphosate (systemic) & Roundup, Glyphosate & broad leaf and grass weeds \\
\hline & fluazifop-p-butyl & Fusilade $^{1}$ & $\begin{array}{l}\text { post-emergence, annual and } \\
\text { perennial grass weeds }\end{array}$ \\
\hline & pelargonic acid & Scythe & broad leaf and grass weeds \\
\hline Repellent & capsaicin & Deer Off & deer, rabbit, mouse repellent \\
\hline \multicolumn{4}{|c|}{$\begin{array}{l}{ }^{1} \text { For use with non-bearing trees only. } \\
2 \text { For nursery/nonbearing trees only. } \\
{ }^{3} \text { For use with dormant trees only. } \\
{ }^{4} \text { Not registered for use in Miami-Dade, Palm Beach, Broward Counties (see label for others). }\end{array}$} \\
\hline
\end{tabular}

Table 14. Pesticides registered for papaya in Florida.

\begin{tabular}{|c|c|c|c|}
\hline Class & Chemical name & Brand name(s) & Pest(s) controlled \\
\hline \multirow[t]{20}{*}{ Insecticides } & pyrethrin + rotenone & Pyrellin & aphids, lepidoptera, thrips \\
\hline & potassium salts of fatty acids & Safer Soap & $\begin{array}{l}\text { aphids, lace bugs, mealy bugs, } \\
\text { spidermites, others }\end{array}$ \\
\hline & spinosad & Spintor & lepidoptera larvae \\
\hline & azadirachtin & Align, Azatin & general insecticide \\
\hline & Beauveria bassiana & Mycotrol & aphids, mealybugs, others \\
\hline & Bacillus thuringiensis & Dipel, others & lepidoptera larvae \\
\hline & malathion & Malthion & thrips, scales \\
\hline & pyrethrins & Pyrenone & aphids, lepidoptera, mites, thrips \\
\hline & fenbutatin-oxide & Vendex & mites \\
\hline & permethrin & Pounce, Ambush & mites \\
\hline & pymetrozine & Endeavor $^{2}$ & whiteflies, aphids, \\
\hline & kaolin (clay) & Surround & barrier and irritant to various insects \\
\hline & Myrothecium verrucaria & DiTera & nematodes \\
\hline & S-methoprene & Extinguish & ants \\
\hline & hydramethylnon & Amdro $^{1}$ & ants \\
\hline & pyriproxyfen & Knack & scales, thrips, ants \\
\hline & bifenazate & Floramite $^{1}$ & mites \\
\hline & fenpropathrin & Tame $^{2}$ & various insects, mites \\
\hline & heythiazox & Savey $^{2}$ & various insects, mites \\
\hline & bifenthrin & Talstar $^{1}$ & various insects, mites \\
\hline
\end{tabular}


Table 14. Pesticides registered for papaya in Florida.

\begin{tabular}{|c|c|c|c|}
\hline Class & Chemical name & Brand name(s) & Pest(s) controlled \\
\hline & pyriproxyfen & Knack, Distance $^{2}$ & \\
\hline \multirow[t]{9}{*}{ Fungicides } & sulfur & $\begin{array}{l}\text { Sulfur 6L, Sulfur Flowable, } \\
\text { Thiolux }\end{array}$ & powdery mildew \\
\hline & copper & Kocide, others & anthracnose \\
\hline & azoxystrobin & Abound & anthracnose \\
\hline & mefenoxam & Subdue Maxx & $\begin{array}{l}\text { Pythium root rot, Phytophthora } \\
\text { root rot }\end{array}$ \\
\hline & chlorothalonil & Bravo & anthracnose \\
\hline & mancozeb & Dithane, Manzate & anthracnose \\
\hline & maneb & Manex & anthracnose \\
\hline & thiabendazole & Decco Salt No. 19 & anthracnose \\
\hline & Trichoderma harzianum & Rootshield $^{2}$ & root system disease protectent \\
\hline \multirow[t]{6}{*}{ Herbicides } & glyphosate (systemic) & Roundup, Glyphosate & broad leaf and grass weeds \\
\hline & oxyfluorfen & Goal & $\begin{array}{l}\text { pre-emergence on broadleaf } \\
\text { weeds }\end{array}$ \\
\hline & diuron & Karmex & annual broadleaf and grass weeds \\
\hline & paraquat & Gramoxone Extra & broad leaf and grass weeds \\
\hline & oryzalin & Surflan & $\begin{array}{l}\text { annual grasses and certain } \\
\text { broadleaf weeds }\end{array}$ \\
\hline & pelargonic acid & Scythe & broad leaf and grass weeds \\
\hline Repellent & capsaicin & Deer Off & deer, rabbit, mouse repellent \\
\hline $\begin{array}{l}{ }^{1} \text { For use wit } \\
{ }^{2} \text { For nurser } \\
{ }^{3} \text { For use wit }\end{array}$ & $\begin{array}{l}\text { n-bearing trees only. } \\
\text { nbearing trees only. } \\
\text { rmant trees only. }\end{array}$ & Sord & 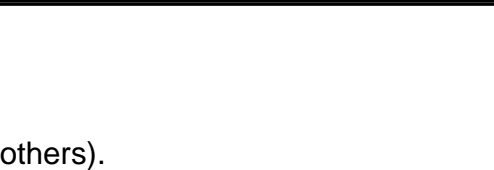 \\
\hline
\end{tabular}

Table 15. Pesticides registered for passion fruit in Florida.

\begin{tabular}{|l|l|l|l|}
\hline \hline Class & Chemical name & Brand name(s) & Pest(s) controlled \\
\hline Insecticides & pyrethrin + rotenone & Pyrellin & aphids, lepidoptera, thrips \\
\hline & potassium salts of fatty acids & Safer Soap & $\begin{array}{l}\text { aphids, lace bugs, mealy bugs, } \\
\text { spidermites, others }\end{array}$ \\
\hline & spinosad & lepidoptera larvae \\
\hline & azadirachtin & Spintor & general insecticide \\
\hline & Beauveria bassiana & Align, Azatin & aphids, mealybugs, others \\
\hline & Bacillus thuringiensis & Mycotrol & lepidoptera larvae \\
\hline & malathion & Dipel, others & thrips, scales \\
\hline & pyrethrins & Malthion & aphids, lepidoptera, mites, thrips \\
\hline & pymetrozine & Pyrenone & whiteflies, aphids, \\
\hline & kaolin (clay) & Endeavor ${ }^{2}$ & barrier and irritant to various insects \\
\hline & S-methoprene & ants \\
\hline & hydramethylnon & Surround & ants \\
\hline & pyriproxyfen & Extinguish $^{1}$ & scales, thrips, ants \\
\hline & bifenazate & Amdro & mites \\
\hline & fenpropathrin & Knack, Distance & various insects, mites \\
\hline & heythiazox & Floramite & various insects, mites \\
\hline
\end{tabular}


Table 15. Pesticides registered for passion fruit in Florida.

\begin{tabular}{|c|c|c|c|}
\hline Class & Chemical name & Brand name(s) & Pest(s) controlled \\
\hline & bifenthrin & Talstar $^{1}$ & various insects, mites \\
\hline \multirow[t]{5}{*}{ Fungicides } & chlorothalonil & Bravo & anthracnose \\
\hline & copper & Kocide, others & anthracnose \\
\hline & azoxystrobin & Abound & anthracnose \\
\hline & mefenoxam & Subdue Maxx ${ }^{2}$ & root rot \\
\hline & Trichoderma harzianum & Rootshield $^{2}$ & root system disease protectent \\
\hline \multirow[t]{3}{*}{ Herbicides } & glyphosate (systemic) & Roundup, Glyphosate & broad leaf and grass weeds \\
\hline & paraquat & Gramoxone Extra & broad leaf and grass weeds \\
\hline & pelargonic acid & Scythe & broad leaf and grass weeds \\
\hline Repellent & capsaicin & Deer Off & deer, rabbit, mouse repellent \\
\hline \multicolumn{4}{|c|}{$\begin{array}{l}{ }^{1} \text { For use with non-bearing trees only. } \\
2 \text { For nursery/nonbearing trees only. } \\
3 \text { For use with dormant trees only. } \\
{ }^{3} \text { Not registered for use in Miami-Dade, Palm Beach, Broward Counties (see label for others). }\end{array}$} \\
\hline
\end{tabular}

Table 16. Pesticides registered for sapodilla in Florida.

\begin{tabular}{|c|c|c|c|}
\hline Class & Chemical name & Brand name(s) & Pest(s) controlled \\
\hline \multirow[t]{16}{*}{ Insecticides } & pyrethrin + rotenone & Pyrellin & aphids, lepidoptera, thrips \\
\hline & potassium salts of fatty acids & Safer Soap & $\begin{array}{l}\text { aphids, lace bugs, mealy bugs, } \\
\text { spidermites, others }\end{array}$ \\
\hline & azadirachtin & Align, Azatin & general insecticide \\
\hline & spinosad & Spintor & lepidoptera larvae \\
\hline & Beauveria bassiana & Mycotrol & aphids, mealybugs, others \\
\hline & Bacillus thuringiensis & Dipel, others & lepidoptera larvae \\
\hline & $\begin{array}{l}\text { various refined horticultural } \\
\text { oils }\end{array}$ & $\begin{array}{l}\text { Sunspray, citrus spray oil, } \\
\text { crop oil, FC 435-66, FC } \\
455-88 \text {, others }\end{array}$ & various aphids, mites, scales \\
\hline & pymetrozine & Endeavor $^{2}$ & whiteflies, aphids, \\
\hline & kaolin (clay) & Surround & barrier and irritant to various insects \\
\hline & S-methoprene & Extinguish & ants \\
\hline & hydramethylnon & Amdro $^{1}$ & ants \\
\hline & pyriproxyfen & Knack, Distance $^{2}$ & scales, thrips, ants \\
\hline & bifenazate & Floramite $^{1}$ & mites \\
\hline & fenpropathrin & Tame $^{2}$ & various insects, mites \\
\hline & heythiazox & Savey $^{2}$ & various insects, mites \\
\hline & bifenthrin & Talstar $^{1}$ & various insects, mites \\
\hline \multirow[t]{2}{*}{ Fungicides } & azoxystrobin & Abound & anthracnose \\
\hline & mefenoxam & Subdue Maxx ${ }^{2}$ & Pythium root rot \\
\hline
\end{tabular}


Table 16. Pesticides registered for sapodilla in Florida.

\begin{tabular}{|c|c|c|c|}
\hline Class & Chemical name & Brand name(s) & Pest(s) controlled \\
\hline & Trichoderma harzianum & Rootshield $^{2}$ & root system disease protectent \\
\hline \multirow[t]{2}{*}{ Herbicides } & glyphosate (systemic) & Roundup, Glyphosate & broad leaf and grass weeds \\
\hline & pelargonic acid & Scythe & broad leaf and grass weeds \\
\hline Repellent & capsaicin & Deer Off & deer, rabbit, mouse repellent \\
\hline \multicolumn{4}{|c|}{$\begin{array}{l}{ }^{1} \text { For use with non-bearing trees only. } \\
{ }_{2} \text { For nursery/nonbearing trees only. } \\
{ }_{3}^{3} \text { For use with dormant trees only. } \\
{ }^{4} \text { Not registered for use in Miami-Dade. } P\end{array}$} \\
\hline
\end{tabular}

Table 17. Pesticides registered for wax jambu in Florida.

\begin{tabular}{|c|c|c|c|}
\hline Class & Chemical name & Brand name(s) & Pest(s) controlled \\
\hline \multirow[t]{15}{*}{ Insecticides } & potassium salts of fatty acids & Safer Soap & $\begin{array}{l}\text { aphids, lace bugs, mealy bugs, } \\
\text { spidermites, others }\end{array}$ \\
\hline & azadirachtin & Align, Azatin & general insecticide \\
\hline & spinosad & Spintor & lepidoptera larvae \\
\hline & Beauveria bassiana & Mycotrol & aphids, mealybugs, others \\
\hline & Bacillus thuringiensis & Dipel, others & lepidoptera larvae \\
\hline & $\begin{array}{l}\text { various refined horticultural } \\
\text { oils }\end{array}$ & $\begin{array}{l}\text { Sunspray, citrus spray oil, } \\
\text { crop oil, FC } 435-66 \text {, FC } \\
455-88 \text {, others }\end{array}$ & various aphids, mites, scales \\
\hline & pymetrozine & Endeavor $^{2}$ & whiteflies, aphids, \\
\hline & kaolin (clay) & Surround & barrier and irritant to various insects \\
\hline & S-methoprene & Extinguish & ants \\
\hline & hydramethylnon & Amdro $^{1}$ & ants \\
\hline & pyriproxyfen & Knack, Distance $^{2}$ & scales, thrips, ants \\
\hline & bifenazate & Floramite $^{1}$ & mites \\
\hline & fenpropathrin & Tame $^{2}$ & various insects, mites \\
\hline & heythiazox & Savey $^{2}$ & various insects, mites \\
\hline & bifenthrin & Talstar $^{1}$ & various insects, mites \\
\hline \multirow[t]{3}{*}{ Fungicides } & azoxystrobin & Abound & anthracnose \\
\hline & mefenoxam & Subdue Maxx ${ }^{2}$ & root rot \\
\hline & Trichoderma harzianum & Rootshield $^{2}$ & root system disease protectent \\
\hline \multirow[t]{2}{*}{ Herbicides } & glyphosate (systemic) & Roundup, Glyphosate & broad leaf and grass weeds \\
\hline & pelargonic acid & Scythe & broad leaf and grass weeds \\
\hline Repellent & capsaicin & Deer Off & deer, rabbit, mouse repellent \\
\hline $\begin{array}{l}{ }^{1} \text { For use witl } \\
{ }^{2} \text { For nursery } \\
{ }^{3} \text { For use witl } \\
{ }^{4} \text { Not register }\end{array}$ & $\begin{array}{l}\text { n-bearing trees only. } \\
\text { nbearing trees only. } \\
\text { ormant trees only. } \\
\text { for use in Miami-Dade, Palm B }\end{array}$ & ch, Broward Counties (see I & or others). \\
\hline
\end{tabular}


Table 18. Pesticides registered for white sapote in Florida.

\begin{tabular}{|c|c|c|c|}
\hline Class & Chemical name & Brand name(s) & Pest(s) controlled \\
\hline \multirow[t]{15}{*}{ Insecticides } & potassium salts of fatty acids & Safer Soap & $\begin{array}{l}\text { aphids, lace bugs, mealy bugs, } \\
\text { spidermites, others }\end{array}$ \\
\hline & azadirachtin & Align, Azatin & general insecticide \\
\hline & spinosad & Spintor & lepidoptera larvae \\
\hline & Beauveria bassiana & Mycotrol & aphids, mealybugs, others \\
\hline & Bacillus thuringiensis & Dipel, others & lepidoptera larvae \\
\hline & $\begin{array}{l}\text { various refined horticultural } \\
\text { oils }\end{array}$ & $\begin{array}{l}\text { Sunspray, citrus spray oil, } \\
\text { crop oil, FC } 435-66 \text {, FC } \\
455-88 \text {, others }\end{array}$ & various aphids, mites, scales \\
\hline & pymetrozine & Endeavor $^{2}$ & whiteflies, aphids, \\
\hline & kaolin (clay) & Surround & barrier and irritant to various insects \\
\hline & S-methoprene & Extinguish & ants \\
\hline & hydramethylnon & Amdro $^{1}$ & ants \\
\hline & pyriproxyfen & Knack, Distance ${ }^{2}$ & scales, thrips, ants \\
\hline & bifenazate & Floramite $^{1}$ & mites \\
\hline & fenpropathrin & Tame $^{2}$ & various insects, mites \\
\hline & heythiazox & Savey $^{2}$ & various insects, mites \\
\hline & bifenthrin & Talstar ${ }^{1}$ & various insects, mites \\
\hline \multirow[t]{3}{*}{ Fungicides } & azoxystrobin & Abound & anthracnose \\
\hline & mefenoxam & Subdue Maxx ${ }^{2}$ & root rot \\
\hline & Trichoderma harzianum & Rootshield $^{2}$ & root system disease protectent \\
\hline \multirow[t]{2}{*}{ Herbicides } & glyphosate (systemic) & Roundup, Glyphosate & broad leaf and grass weeds \\
\hline & pelargonic acid & Scythe & broad leaf and grass weeds \\
\hline Repellent & capsaicin & Deer Off & deer, rabbit, mouse repellent \\
\hline \multicolumn{4}{|c|}{$\begin{array}{l}{ }^{1} \text { For use with non-bearing trees only. } \\
{ }^{2} \text { For nursery/nonbearing trees only. } \\
{ }^{3} \text { For use with dormant trees only. } \\
{ }^{4} \text { Not registered for use in Miami-Dade, Palm Beach, Broward Counties (see label for others). }\end{array}$} \\
\hline
\end{tabular}

